\title{
ANALYSIS OF THROUGHPUT AND EFFICIENT BLOCK SIZE FOR MULTIMEDIA TRAFFIC USING IEEE 802.11 WIRELESS LANS
}

\author{
Shamganth . $K^{1}$ and Murali Mohan Reddy . $\mathbf{M}^{2}$ \\ Vellore Institute of Technology, Vellore,India \\ E-mail : 'kshmganth@vit.ac.in, ${ }^{2}$ murali221423@gmail.com
}

\begin{abstract}
As the demand for broadband multimedia wireless services increases, the need to provide QoS and higher data rate enhancements to the widely deployed IEEE 802.11 wireless LAN has become apparent. However, currently deployed IEEE 802.11 standards have little or no QOS support functions built in to the MAC layer. The IEEE 802.11e EDCF mechanism is currently being developed to provide QoS functions in the MAC layer based on service differentiation and traffic class prioritization. Along with the $802.11 \mathrm{e}$ QoS amendment, 802.11n defines advanced ARQ policies designed to increase the channel utilization efficiency by reducing MAC protocol overhead. Although these techniques provide vast improvements over the legacy 802.11 protocol, opportunities exist with in this framework to optimize performance based on the type of traffic being serviced. In this paper a distributed medium access scheme called EDCF, which is adopted in an upcoming standard IEEE 802.11e to allow prioritized medium access for applications with QoS requirements is analyzed and its performance is evaluated using OPNET Modeler. We also investigate the performance optimization of the 802.11 Block Ack ARQ policy, and discuss adaptive block size algorithm for throughput improvement.
\end{abstract}

Key words: $8 \quad 0 \quad 2$. ARQlop'timization; Block ACK; WLAN;HCF;EDCF;OPNET Modeler

\section{INTRODUCTION}

Wireless access has been increasingly popular recently due to portability and low cost. Extensive research is being carried out to increase provided data rates of wireless networks to values comparable with wired networks. The emerging technologies for wireless local area networks (WLANs) are defined by the IEEE 802.11 standards, which started by $802.11 \mathrm{~b}$ with physical layer (PHY) data rates of up to $11 \mathrm{Mbps}$ and were enhanced in $802.11 \mathrm{a} / \mathrm{g}$ to provide up to $54 \mathrm{Mbps}$ with the introduction of Orthogonal Frequency Division Multiplexing (OFDM)[1]. Along with the 802.11e QoS amendment [2],current standardization efforts within the 802.11 work group include $802.11 n$ - an

advanced, high-rate physical (PHY) and medium access control (MAC) layer specification designed to offer PHY layer throughputs up to $600 \mathrm{Mb} / \mathrm{s}$, with in excess of $100 \mathrm{Mb} / \mathrm{s}$ experienced at the MAC layer [3].

Although the 802.11 Medium Access Control (MAC) sub layer provides limited QoS support for multimedia traffic through the optional centralized access mechanism called the Point Coordination Function (PCF), further QoS enhancements are needed in order for 802.11 WLANs to support the increasing demand for multimedia applications both in infrastructure mode (incorporating at least one central access point connected to a wired network) and ad hoc or peer-to-peer architecture. The PCF possesses several problems in providing guaranteed QoS policies, including unknown transmission durations of polled stations, and unpredictable beacon delays, both of which may severely affect QoS-sensitive applications as unpredictable time delays are introduced [4]. As the predominant WLANs in use today and those expected to be use in future use decentralized or distributed medium access mechanisms based on carrier sensing [5], further enhancements to the 802.11 distributed access mechanism called the Distributed Coordination Function (DCF) are also required in order to provide QoS provisions for multimedia applications. The DCF is a mandatory part of the IEEE 802.11 MAC standard, and offers best effort packet transmission services. In order to support QoS in 802.11 WLAN, the upcoming IEEE 802.11e standard adds a new function called a hybrid coordination function (HCF) that includes both controlledcontention-free and contention-based channel access methods in a single channel access protocol. The HCF uses a contentionbased channel access method called enhanced DCF (EDCF) that operates concurrently with a controlled channel access mechanism based on a central polling mechanism. HCF supports both prioritized and parameterized medium access. Fig. 1 shows the architecture of $802.11 \mathrm{e}$ and its coexistence with legacy 802.11 This paper will briefly review the main features and functions of the 802.11e standard[6]. The detailed discussion will be focused on EDCF function. A comparison between DCF and EDCF is also given.

In this paper we also discuss the distributed, adaptive block size algorithm[7] for multimedia traffic, where the Block Ack protocol's block size is dynamically adjusted based on traffic class requirements, link state information and transmission channel characteristics. 


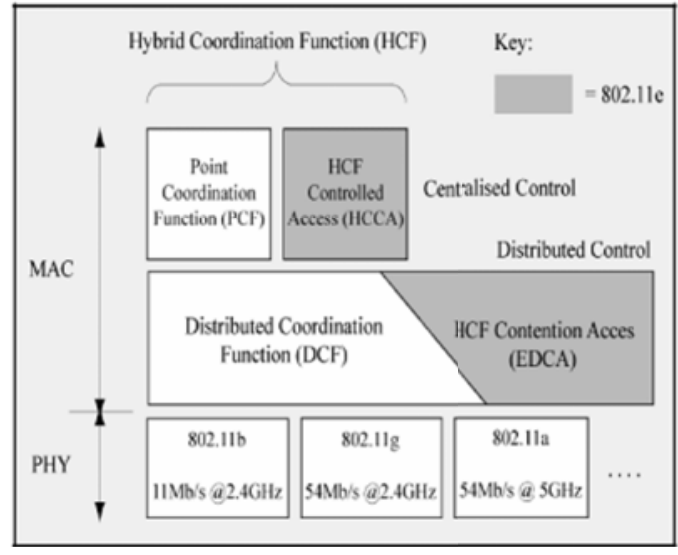

Fig 1. 802.11e Architecture

The rest of the article is structured as follows; section II provides an overview of the 802.11 MAC. Section III provides an overview of the advanced $A R Q$ policies used in $802.11 \mathrm{e} / \mathrm{n}$. section IV describes the adaptive block size algorithm. The simulation model used to analyze prioritized medium access for applications with QoS requirements and $A B S$ algorithm along with results is presented in section $\mathrm{V}$,. A conclusion is drawn in section VI.

\section{OVERVIEW OF 802.11 MAC}

In 802.11 access layer there are two sub-layers: Logical Link Control (LLC) and Medium Access Control (MAC). IEEE 802.11 specifies the transmission schemes of PHY layer and the protocol that MAC layer follows. This paper focuses in the MAC sub-layer.

The time between two MAC frames is called interframe space (IFS). IEEE 802.11 defines four different IFSs. Short Inter Frame Space (SIFS), Point Coordination Function Inter Frame Space (PIFS) and distributed Coordination Function (DIFS) are used under normal conditions and represent three different priority levels for medium access. The shorter the IFS, the higher priority in medium access. The fourth IFS, called Extended Inter Frame Space (EIFS), is used when a station detects an ongoing transmission as being interfered. In the following, the durations used in 802.11 are listed in order, from shortest to longest.

- aSlot Time: The duration aSlot Time is used to calculate the IFSs. SIFS and aSlot Time are the basis of all other durations.

- SIFS: The SIFS is used to prioritize the immediate Acknowledgement (ACK) frame of a data frame, and different control frames.

- PIFS: The PIFS is used by stations operating under the PCF to obtain channel access with highest priority. PIFS is calculated as: PIFS = SIFS + aSlot Time
- DIFS: The DIFS is used by stations operating under the DCF to obtain channel access to initiate frame exchanges. DIFS is calculated as: DIFS = SIFS + 2.aSlot Time.

- EIFS: The EIFS is used instead of DIFS by stations operating under the $\mathrm{CF}$ whenever the $\mathrm{PHY}$ indicates that a frame transmission did not result in a correct sequence.

\section{A. 802.11 DCF}

The DCF is the mandatory 802.11 MAC mechanism, and is based on the distributed Carrier Sense Multiple Access with Collision Avoidance (CSMA/CA) protocol. The reason for collision avoidance as compared to the Collision Detection (CSMA/CD) in 802.3 (Ethernet) networks is that collisions are extremely hard to detect in a wireless medium, and therefore prevention to the maximum extent possible is a recommended approach. A station (STA) operating under DCF can transmit after it senses the medium to be idle for a defined time period called the DCF Inter- Frame Space (DIFS). If the medium is found busy, a STA defers access until the end of the current transmission. After deferral, the STA waits another DIFS interval and then selects a random backoff interval drawn from a uniform distribution over the range of 0 to Contention Window (CW), decrementing a backoff interval counter when the medium is idle. The STA can begin to transmit on expiration of this counters shown in Fig.2. if a transmission is successful, the CW value is doubled and a new backoff procedure is started. This process continues until the successful acknowledgement of a MSDU, or a retransmission attempt limit is reached upon which the MSDU is discarded.

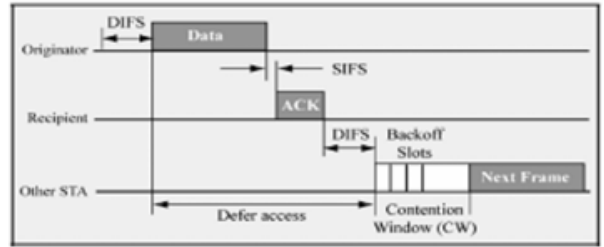

Fig. 2. 802.11 DCF Timing Operation

The CW is an integer number of slots, where one slot has a duration related to the PHY layer characteristics being used, e.g. $9 \mu \mathrm{s}$ for $802.11 \mathrm{a}$. CW is initially equal to Cwmin and is increased exponentially upon every retransmission attempt until reaches CWmax, where it remains until it is reset. The backoff procedure is used to reduce the probability of collisions, while the increasing of CW size for successive retransmission attemts improves the stability of the access protocol under high load conditions. The current 802.11 DCF does not provide any QoS provisions, as all STAs use the same CSMA/CA access parameters to contend for the channel, and therefore have the same priority. 


\section{B.802.11e EDCA}

The IEEE 802.11Task Group e (TGe) is defined Quality of Service (QoS) enhancements to the 802.11 $M A C$, with a distributed access mechanism called Enhanced Distributed Channel Access (EDCA). In order to provide service differentiation, faster access to the channel must be given to a Traffic Category (TC) with a higher priority. Proposals presented by TGe bind the priority of a TC to channel access by assigning different CSMA/CA access parameters for each TC, as shown in Fig. 3. These include the inter- frame space called an Arbitration Inter-Frame space (AIFS), and the parameters that regulate the CW size, being Cwmin, CWmax, and Persistence Factor (PF). The PF indicates the scaling factor used in computing the new CW size on every unsuccessful attempt to transmit. For each retransmission a new CW value is used calculated by the following formulae:

$$
\text { Cwnew }=(\operatorname{PFx}(\text { CWold }+1)) ? 1
$$

Each backoff entity with in the stations contends for a TXOP independently. It starts down-counting the backoff counter after detecting the channel being idle for an Arbitration Inter Frame Space Duration (AIFSD [AC]). The AIFSD [AC] is at least PIFS, and can be enlarged per AC with the help of the parameter Arbitration Inter Frame Space (AIFS [AC]). The AIFS [AC] defines the duration of AIFSD $[A C]$ according to

AIFSD $[A C]=$ SIFS + AIFS $[A C]$. aSlot Time, where 1 $\$$ AIFS $[A C] \leqslant 10$ priority.

The smaller AIFS [AC], the higher the channel access

Another EDCF parameter is the minimum size of the contention window, CWmin [AC], and is dependent on the AC. The initial value for the backoff counter is a random number taken from an interval defined by the contention window (CW), similar to legacy DCF. The contention window may be the initial minimum size $\mathrm{CWmin}[\mathrm{AC}]$, or higher values. In case packet transmission failures occurred, entity selects its counter as random number drawn from the interval [1, CW +1]

Each TC has its own queue, and contends for the medium using a separate CSMA/CA instance. Collisions between TCs with in the same STA are resolved by granting access to the $\mathrm{TC}$ with the highest priority, with the lower priority TC behaving as if there was an external collision on the wireless medium [8].

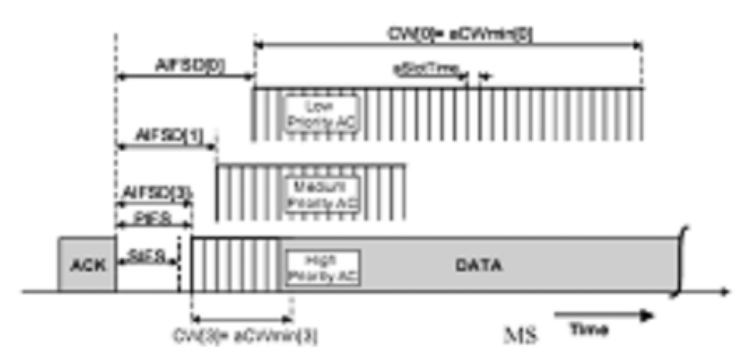

Fig 3. 802.11e EDCA Timing Operation

Fig. 4 shows the implementation model architecture of the mapping from higher layers into TC queues, each with their own channel access functions and virtual internal collision resolution. Once a STA has gained access to the medium, it can initiate a Transmission Opputunity (TXOP), which is a bounded duration time interval in which the STA may perform frame bursting where a sequence of SIFSseperated data and ACK frames are exchanged.

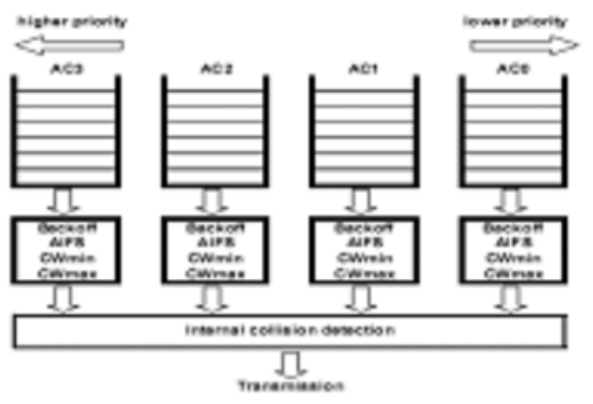

Fig 4. EDCA Implementation Architecture

The Typical values of $C W$ limits and AIFS for different ACs in the QoS parameters set is shown in Table 1.

Table 1. Typical QoS parameters

\begin{tabular}{|l|l|l|l|}
\hline $\mathbf{A C}$ & $\mathbf{C W m i n}$ & $\mathbf{C W m a x}$ & AIFS \\
\hline 0 & CWmin & CWmax & 2 \\
\hline 1 & CWmin & $C W \max$ & 1 \\
\hline 2 & $(\mathrm{CW} \min +1) / 2-1$ & $\mathrm{CW} \min$ & 1 \\
\hline 3 & $(\mathrm{CW} / \min +1) / 4-1$ & $(\mathrm{CW} \min +1) 2-1$ & 1 \\
\hline
\end{tabular}

The priority to access category mappings and different CSMA/CA access parameters for each TC which are used in the simulation is shown in below Table 2: 
Table 2: Priority to access category mappings and differentCSMA/CA access parameters for each TC

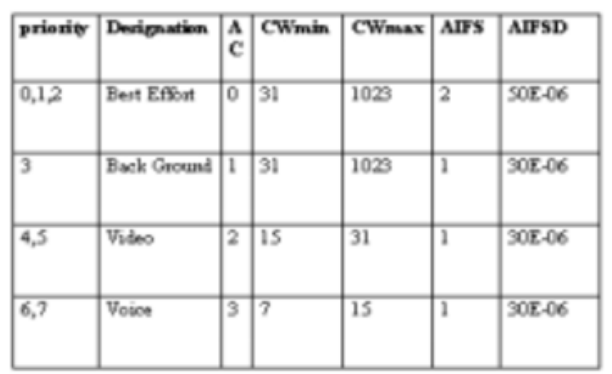

\section{ARQ POLICIES}

The 802.11e standard supports three different ARQ policies, namely Ack (traditionally stop and wait), No Ack, and Block Ack (selective repeat). The legacy 802.11 WLAN standard supports only the Ack policy, which is characterized by the transmission of a positive acknowledgement (Ack) by a recipient node to the originating node upon the successful reception of a data packet, also known as a MAC Protocol Data Unit (MPDU). Acknowledgement of a MPDU occurs after SIFS duration. If a MPDU is received in error or the transmission of the Ack is unsuccessful, the originating node retransmits the packet. If the packet has not been successfully transmitted after a predefined number of attempts, it is discarded. When operating using a No Ack policy, MPDUs is transmitted once and only once regardless of whether they are received in error or not at all. The Block Ack mechanism defined in the 802.11e allows a group of MPDU $s$ to be transmitted consecutively within a transmission opportunity (TXOP), while bundling all corresponding acknowledgements into single collective Ack. A TXOP is defined as a bounded duration in which a node may perform packet bursting; where a sequence of SIFS separated packets are exchanged. Block Ack is initialized by an exchange of setup frames that negotiate parameters including buffer size and Block Ack type, where the Block Ack type is either immediate or delayed. Fig. 5 shows the immediate Block Ack policy. After initialization, the originator may transmit a sequence of SIFS separated MPDU s, with the total number of MPDU s (block size) not exceeding the buffer size negotiated at the setup. If the RTS/CTS protection mechanism is not used, an Ack must be transmitted after the first MPDU in the TXOP, as illustrated in Fig. 2a. The originating node requests an Ack of outstanding MPDU $s$ by sending a Block Ack Request (BAR) frame. If immediate Block Ack is used, the recipient responds by transmitting a Block Ack (BA) frame immediately after the BAR, which contains the acknowledgement state of data packets received from the originator. ABA frame can acknowledge up to 64 MSDU s, with each MSDU fragmented up to 16 times. Delayed Block Ack allows a node to acknowledge the BAR with an ACK, then prepare a BA response and send it in the earliest possible subsequent TXOP. The originator acknowledges the receipt of the BA with an ACK. This delayed approach is primarily intended to facilitate inexpensive implementations that use the processing power of a host, as well as to allow existing implementations to use this feature with minimal hardware changes. In the event of unsuccessful transmission, packets are retransmitted in the next available TXOP and acknowledged as soon as possible, as shown in Fig. 5c.

This process continues until they are discarded from the transmitter queue upon expiry of an access category (AC) specific delay threshold called a MSDU Lifetime. A timer is started when a MSDU is first passed to the MAC layer, and if it exceeds its associated MSDU Lifetime before successful transmission, all remaining fragments of that MSDU are discarded by the originating node without any further attempt to complete delivery. This is advantageous for multimedia traffic, as if a multimedia data packet is not transmitted within a certain delay; it often exceeds its effective usefulness or ability to contribute at the receiving application, therefore consuming unnecessary bandwidth when transmitted.

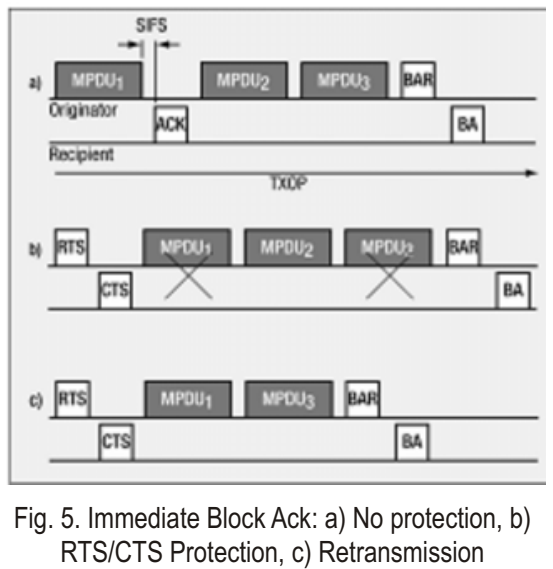

\section{ADAPTIVE BLOCK ACK}

The Adaptive Block Size algorithm, here on in referred to as $A B S$, aims to optimize throughput by dynamically adapting the block size (Bs), based on traffic requirements, link state information, and transmission channel conditions. We define the set of Block Ack (BA) $\mathrm{ARQ}$ strategies as

$$
A R Q=\{B A 0, B A 1, \cdots \cdots B A 64, B A \propto
$$

Where $\mathrm{BA} 0 \equiv \mathrm{ACK}, \mathrm{BA} \infty \equiv$ No Ack, and BA1..64 $\equiv$ Block Ack1..64 
The ABS algorithm's aim, as expressed in equation (3), seeks to find the optimal $A R Q$ strategy that maximizes throughput for different traffic categories $\mathrm{i}$, under varying instantaneous channel conditions $x=$ (SNR, contention).

$$
\begin{aligned}
& \text { ARQ opt }(i, x)=\arg \max \text { Throughput }(A R Q(i, x)) \\
& \text { Bs? }[0,1, \cdots .64, \infty
\end{aligned}
$$

This strategy results in the best link throughput subject to the following delay and packet loss (PL) constraints:

Delay $(A R Q(i, x)) \leqslant M S D U$ Lifetime ,

$\mathrm{PL}(\mathrm{ARQ}(\mathrm{I}, \mathrm{x}) \lessgtr \mathrm{PLthreshold}$

Fig. 6 shows a timing diagram of the ABS algorithm. The algorithm is an originator initiated scheme, in that no additional feedback other than that provided through normal protocol operation is used to provide feedback to the originator. This feedback along with link state information from the originator is used to intelligently steer the block size to provide optimal throughput. A packet loss rate metric is used to determine when to change $B s$, calculated using information from the BA and MSDU Lifetime discard rate. Upon reception of a BA, the number of successfully transmitted MSDUs is extracted from the BA and added to a counter. Likewise, when a MSDU is discarded from the transmission queue due to MSDU Lifetime expiry, a counter representing the number of discarded MSDUs is incremented. The originator periodically calculates $\mathrm{PL}$ using these values, and if found to be above or below a traffic class dependant range (Dead-band), the ABS algorithm dynamically adapts Bsaccordingly. This control strategy is illustrated in Fig. 7, where Bs is increased for PL values less than the deadband, and reduced when PL is greater than the deadband.

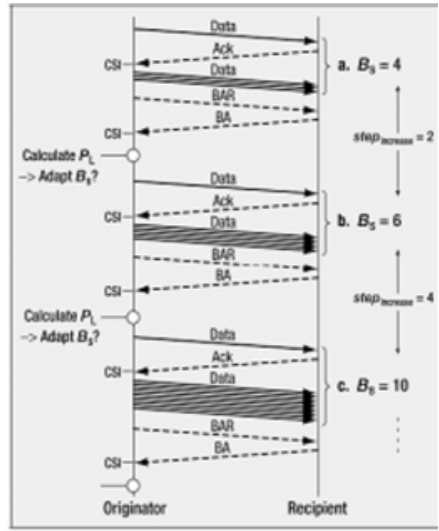

Fig. 6. ABS algorithm timing diagram

Channel state information (CSI), such as received signal strength indicator (RSSI) or SNR, is used to determine how much Bs is changed (step size) in each increment. If the channel conditions are found to have diverged by a differential amount (SNR_DIFFERENTIAL) since the last channel assessment, the step size is changed using a binary exponential scheme, i.e. the step size used when increasing the block size is doubled upon improving channel conditions, and halved if the channel is found to be degrading. Conversely, the step size used to reduce the $B s$ is doubled on channel degradation, and halved on channel improvement. This is best illustrated in Fig. 6a, where Bs has an initial size of 4 , and the step size is initially set to 2 . If a periodic PL calculation shows that an increase in Bs is favorable, Bs is increased by the step size to a value of 6 (Fig. $6 \mathrm{~b}$ ). If CSI feedback shows that channel conditions are improving, the step size is doubled to a value of 4 , thus causing $B$ s to be increased to a value of 10 upon the next favorable periodic PL calculation (Fig. $6 c)$. As well as the normal operating bounds of Bs imposed by the BlockAck $A R Q$ policy, an upper and lower block size limit (BS_MAX, BS_MIN) can be specified, restricting the ABS algorithm from changing the value of Bs beyond these bounds. The originator is able to gain CSI through mechanisms such as RSSI, closed-loop modulation and coding scheme (MCS) adaptation [9], or customized feedback through control frames such as CTS, ACK and BA. As this feedback can occur more frequently than the packet loss rate calculation, the step size is used as a fine grained control in the algorithm.

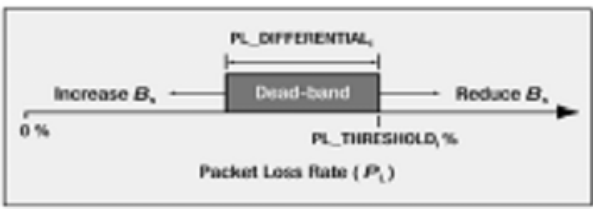

Fig. 7. ABS Algorithm Control Strategy

\section{SIMULATION EVALUATION}

\section{A. 802.11e EDCF}

A simulation model was constructed using OPNET Modeler. In the simulation, four IEEE 802.11 wireless stations with EDCF mechanisms were configured in ad hoc mode shown in Fiq. 8.

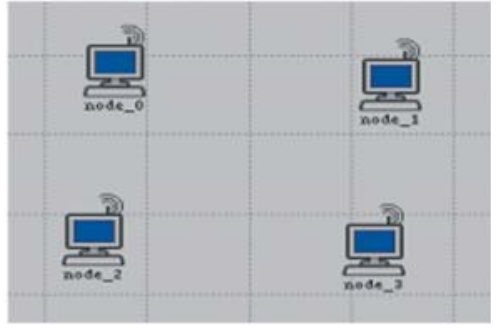

Fig .8 The Simulation Scenario

The WLAN Node Model and MAC_HCF Process model is shown in below Fig 9, Figu 10 respectively. 
Four stations remain stationary during the simulation. The simulation uses a standard $802.11 \mathrm{~b} \mathrm{PHY} \mathrm{module} \mathrm{with}$ maximum data rate up to $11 \mathrm{Mbps}$ to simulate the wireless medium. All physical characteristics was according to 802.11b direct sequence spread spectrum (DSSS) PHY parameters which is shown in Fig 11, in which Cwmin $=31$, CWmax $=1023$, SIFS Time $=10 \mu$ sand Slot Time $=20 \mu$ shown in Fig 12.

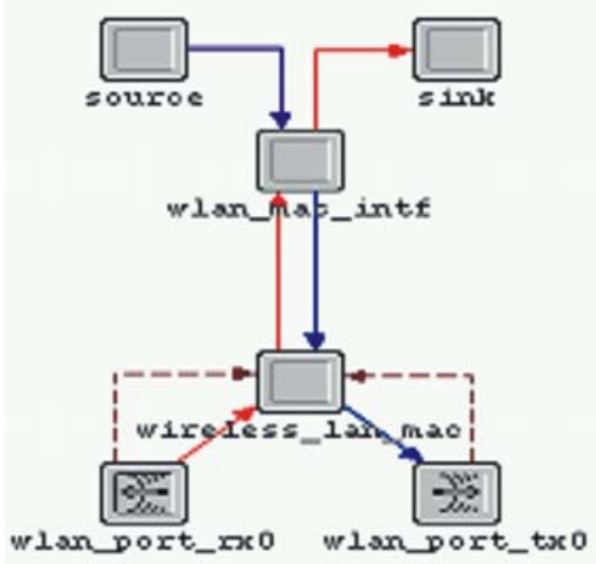

Fig 9: WLAN Node Model

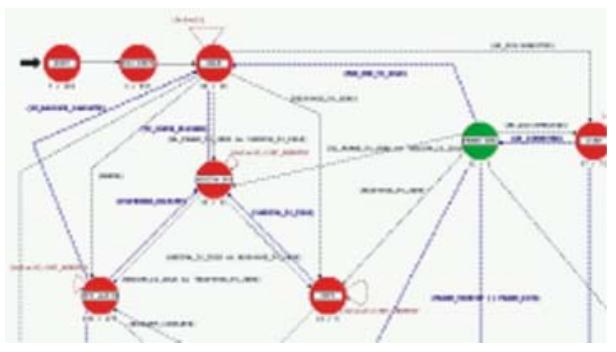

Fig 10: Process Model for WLAN MAC_HCF

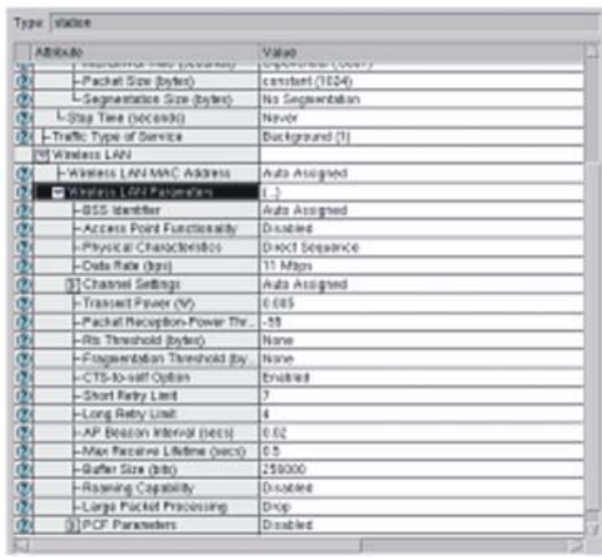

Fig .11 Wireless LAN parameters

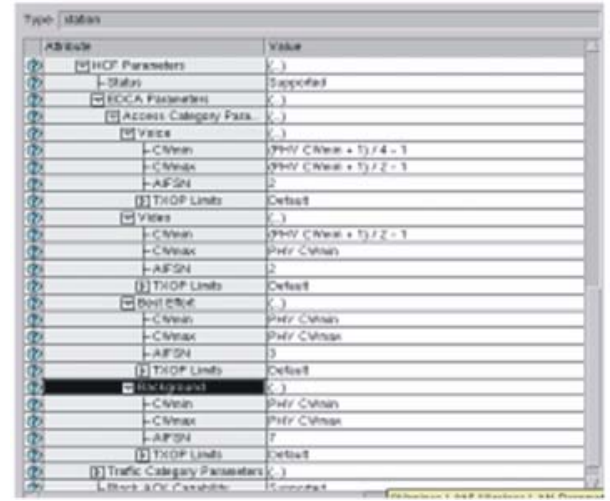

Fig . 12 EDCA Access Category Parameters

All four traffic classes were fed into the MAC layer from the higher layer; these corresponded to $\mathrm{AC}(0), \mathrm{AC}(1)$, $A C(2)$ and $A C(3)$, respectively. In the simulation we assumed that each traffic class has an equal portion of the total data traffic in terms of average number packets generated per unit time. The packets had the same size of 1024 bytes and remainded constant during the simulation. The packets of $A C(0), A C(1)$, and $A C(2)$ were generated according to exponential process with a mean inter-arrival time equal to $0.0001 \mathrm{~s}$ shown in Fig 13 , while $\mathrm{AC}(3)$ packets were generated at a constant rate to simulate a voice source.

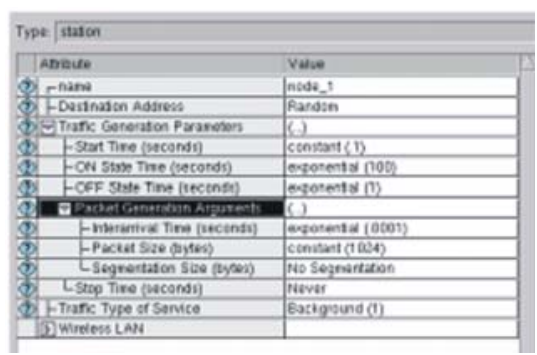

Fig 13 Traffic Generation Parameters

Figure 14 shows the average medium access delays for different ACs in the EDCF mechanism. As shown, $A C(3)$ has the smallest average medium access delay, $\mathrm{AC}(0)$ the largest. The horizontal coordinate represents the simulation time in minutes.

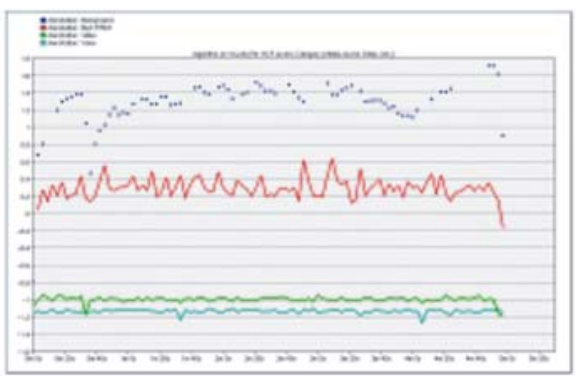

Fig 14. Medium Access Delay for Different Acs 


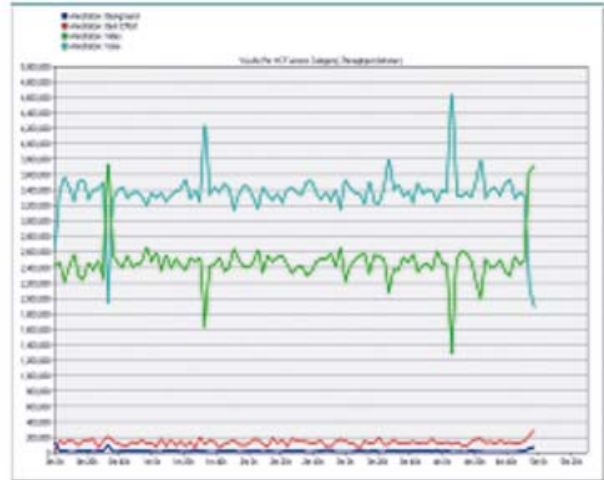

Fig 15. Throughputs for Different Acs

In Fig.15 the throughput for different ACs over the WLAN are shown. We can see that $A C(3)$ has the highest value of throughput, while the throughput of $A C(0)$ is lowest. These results are as expected since the EDCF differentiates the traffic classes and supports priority access. Thus, the higher-priority traffic categories have a smaller medium access delay and more bandwidth.

\section{B.ABS}

A comprehensive discrete event simulation model using OPNET Modeler is used to analyze the performance of the ABS algorithm. Fig.16 shows a node model of the ABS.

The src, rt_1, and rr_1 processors are pre-existing OPNET processors that generate, transmit and receive packets respectively. Packets are generated in the s'c' processor at a data rate of 9600 and sent to the saw processor, which contains the process model. These packets are received in to the sáw' processor via the source input stream. The rt__' ${ }^{\prime}$ and rr'_ $^{\prime}{ }^{\prime}$ processors then transmit or receive packets at the project level

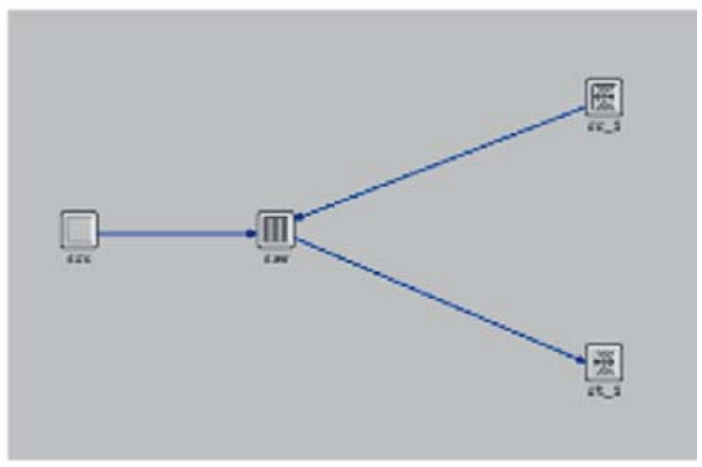

Fig. 16 Node Model for ABS Algorithm

In the simulation two nodes were configured in adhoc mode as shown in Fig. 17. tx acts as an transmitter and rx acts as an receiver

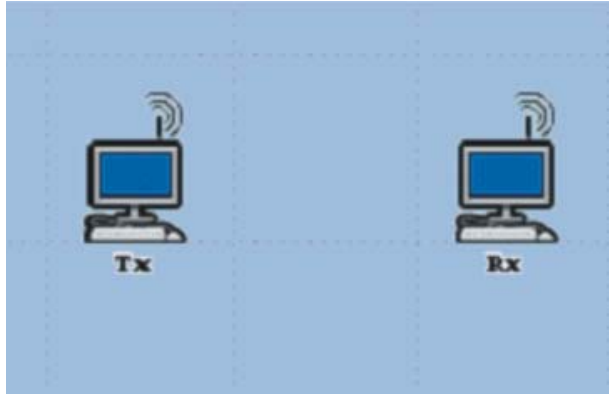

Fig.17 Network Model for ABS Algorithm

Fig. 18 and Fig. 19 a process modeled as a FSM(Finite State Machine) which are placed in the sáw' processor of the transmitter(tx) and the receiver(rx) respectively. We can see three separate states; init, IDLE and ACK Wait. The states are linked together by transitions, which may be conditional or unconditional.

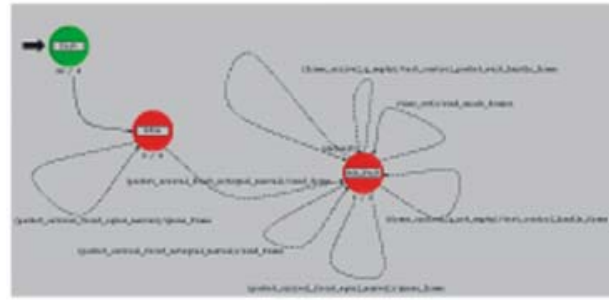

Fig.18 Process Model for Transmitter(tx)

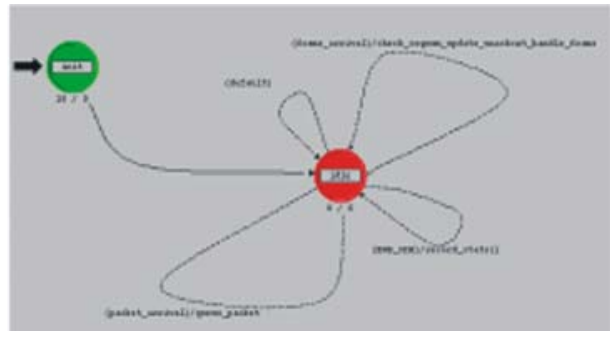

Fig. 19 Process Model for Receiver(rx)

Conditional transitions are those that require a predefined condition to become true before the transition can take place. Unconditional transitions don't require any condition to be true befote the transition can take place. The condition to be fulfilled for each transition is contained with in the curved brackets ().Along with the condition for each transition, there is a transition executive shown after the I"symbol, which is a block of code that executes each time the specified transition takes place.As an example take the ('packet_arrival_ frame_ equal_ maxval)/queue_frame' condition and executive shown in Fig. 18. The condition to be fulfilled here is pácket_arrival_frame_equal_maxval', which is defined in OPNET's header block and the transition executive is qúeue_frame' defined in OPNET's function block. 
With in the header block in OPNET, any conditions associated with the process model are defined, as well as the definitions of any source streams or transition/ executive streams. The function block is an area set aside for any code that describes the operations of any transition executives associated with that process model. $C$ code may also be attached to the Enter or Executives, of the various states with in the process model. If code is placed with in these Enter or Exit executives, this code will always be executed when entering or existing from the associated state.

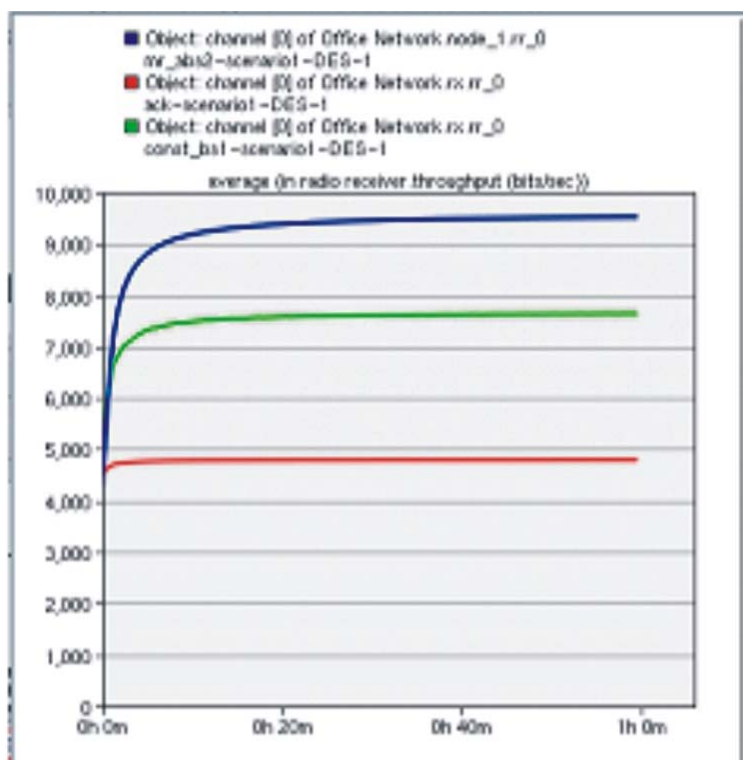

Fig. 20 Throughput Comparison

Fig. 20 shows the throughput achieved when using ACK, Block ACK, and Block ACK with ABS enabled. As expected, Block ACK configured with a static block size of 4 , out performs the legancy $A c k A R Q$ policy due to reduced control overhead and aggregated acknowledgements. As the ABS algorithm is able to dynamically adjust the block size based on channel conditions, larger block sizes are able to be employed when suitable, thus providing superior throughput over a static Block ACK Policy and the legancy ACK mode.

\section{CONCLUSION}

A detailed EDCF and HCF medium access mechanism of the IEEE 802.11e standard is presented in this paper. The EDCF for QoS support was evaluated. The simulation results show that EDCF works well for differentiated data services and priority access to the medium. We also consider the performance optimization of the 802.11 Block Ack ARQ policy, and discuss adaptive block size algorithm for throughput improvement.
Although the No ACKARQ policy is the most efficient in regards to protocol overhead, there is a trade-off in reliability, as data packets are transmitted only once regardless of their error status. Fortunately, multimedia traffic can often cope with a small loss ratethrough techniques such as error error resilent source coding, forward error correction (FEC), and error concealment schemes at the receiver. The ABS algorithm exploits this characteristic, enabling an oppotunity for throughput enhancement and efficient channel utilization when transmitting multimedia traffic.

\section{REFERENCES}

[1] IEEE 802.11a-1999, 1999. P'Part 11: Wireless LAN Medium Access Control (MAC) and Physical Layer (PHY) Specifications High-Speed Physical Layer in the $5 \mathrm{GHZBand"} \mathrm{.}$

[2] IEEE Std 802.11e -2005, Amendment to IEEE Std 802.11, 1999 Edition (Reaff 2003), Amendment 8: Medium Access Control (MAC) Quality of Service Enhancements.

[3] Enhanced Wireless Consortium (EWC), H"T MAC Specification", Versionv1.24, http://www.enhancedwirelessco nsortium.org

[4] Mangold, Choi, May, Klein, Hiertz, Stibor, IEEE 802.11e Wireless LAN for Quality of Service".

[5] Pattara-Atikom, Krishnamurthy, Banerjee, June 2003. Distributed Mechanisms for Quality of Service in Wireless LANs", IEEE Wireless Communications, pp. 26-34.

[6] Gu, Zhang, June 2003, Q’oS Enhancements in IEEE802.11 Wireless Local Area Networks", IEEE Communications Magazine, pp. 120-124

[7] J. Wall, J. Khan, 2007. Towards Optimal Multimedia Packet Bursting for IEEE 802.11 Wireless LANs", IEEE communication society, proceedings,pp. 2151-2156

[8] Gu, Zhang, June 2003, Q'oS Enhancements in IEEE802.11 Wireless Local Area Networks", IEEE Communications Magazine, pp. 120-124

[9] 2007 Towards Optimal Multimedia Packet Bursting for IEEE 802.11 Wireless LANs Joshua Wall, Jamil Khan, school of electrical engineering \& computer science, university of Newcastle, Australia, IEEE proceedings 
[10] Wireless Local Area Networks" by Benny Bing, Ajohn Wiley \& sons.inc., publication

[11] Data and Computer Communications by William stallings

[12] OPNET Technologies Modeler Documentation

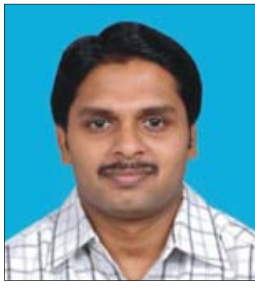

Mr. K. Shamganth received his Master of Engineering degree in Communication systems from G.C.T, Coimbatore, Anna University. He is currently working as a Senior Lecturer in V.I.T University, Vellore, TamilNadu. His research interests include Wireless Technologies: WLAN 802.11a/b/n,WiMax, VoWLAN ;Wired Technologies:VOIP and Protocols: SIP, RLC,MAC,CC

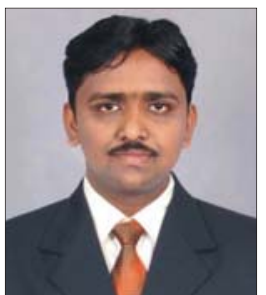

Mr. M. Murali Mohan Reddy received his Bachelor of Science Degree in Maths, Electronics and Computer science from S.V.K.P \& Dr.K.S Raju Arts \& Science College, Penugonda affiliated to Andhra University and Master of Science in Electronics from GVP P.G College, affiliated to Andhra University, Vishakhapatnam and currently pursuing Master of Technology degree in Communication Engineering from VIT University, Vellore. 